\title{
Efecto de marco en un juego de coimas: La relevancia de la dimensión moral
}

\section{Frame effect in a bribery game: The relevance of the moral dimension}

\author{
Carlos Maximiliano Senci ${ }^{1}$, Rodrigo Moro ${ }^{2}$ y Esteban Freidin ${ }^{3}$
}

${ }^{1}$ Doctor en Filosofía. Magister en Derecho. Becario postdoctoral en el Instituto de Investigaciones Económicas y Sociales del Sur (IIESS). Asistente de Docencia en la Universidad Nacional del Sur, Bahía Blanca, Prov. de Buenos Aires, Argentina. E-mail: maximiliano.senci@uns.edu.ar

${ }^{2}$ Doctor en Filosofía. Investigador adjunto del Consejo Nacional de Investigaciones Científicas y Técnicas (CONICET) en el Instituto de Investigaciones Económicas y Sociales del Sur (IIESS).

Profesor en la Cátedra de Filosofía de la Ciencia en la Universidad Nacional del Sur.

${ }^{3}$ Licenciado en Psicología. Doctor en Zoología. Investigador independiente del Consejo Nacional de Investigaciones Científicas y Técnicas (CONICET) en el Instituto de Investigaciones Económicas y Sociales del Sur (IIESS).

Los autores agradecen la colaboración de Marcelo Sapognikoff durante la realización del experimento reportado en este artículo.

Instituto de Investigaciones Económicas y Sociales del Sur (IIESS).

Bahía Blanca, Provincia de Buenos Aires. Argentina.

\section{Resumen}

La corrupción es un fenómeno que involucra componentes morales. Sin embargo, este aspecto no se ha visto reflejado en los experimentos de corrupción de manera inequívoca. De los elementos utilizados para señalizar la presencia de normas, tanto las externalidades negativas como el castigo y la introducción de marcos, han arrojado resultados mixtos o nulos, lo que pareciera cuestionar su validez como instrumentos para estudiar la corrupción.

En este experimento se buscó evidencia de que el componente moral está presente en un juego de coimas. Se contó con dos grupos independientes de participantes $(\mathrm{N}=106)$ que fueron expuestos al juego en un marco neutro o con opciones que llevaban etiquetas con contenido semántico concreto (por ejemplo, ofrecer un trato corrupto). Por último, para evaluar la connotación moral de los escenarios de toma de decisiones, se solicitó a los participantes que completaran (post-decisión) una escala de maquiavelismo, que ha estado asociada a diferentes aspectos en la toma de decisiones morales. El experimento logró identificar un claro efecto de marco tanto para los jugadores A como para los B. Los resultados muestran que los participantes eligieron las opciones corruptas en menor medida en el tratamiento con el marco cargado que con el marco neutro. Además, las respuestas al juego mostraron diferencias individuales en el nivel de maquiavelismo, ya que puntajes elevados de maquiavelismo estuvieron directamente asociados a ofertas corruptas, aunque solo en el marco cargado.

Palabras clave: Corrupción; Marco; Economía experimental; Moral; Externalidades. 


\section{Abstract}

Corruption is a phenomenon that involves moral components. Despite the general agreement about the involvement of a moral transgression in the characterization of corruption, it has been difficult to find clear evidence of the implication of the participant's morality in their choices in bribery games. To signal the presence of norms, researchers have relied on negative externalities, punishment and frames, but they have yielded mixed or null results. In a bribery game framed in a neutral way Abbink, Irlenbusch and Renner (2002) showed that the participants were sensitive to the possibility of receiving sanctions, but not to produce negative externalities on the other participants in the session. The insensitivity to increasing degrees of externalities was also the result obtained by Barr and Serra (2009) in their neutral version of a single round bribery game. According to these results, games with abstract or neutral frames do not seem to have the aforementioned moral dimension, which questions their validity as instruments to study corruption. This study searched for evidence of a moral component in a bribery game. The experiment relied on two independent groups of participants $(\mathrm{N}=106)$ who played the game either framed in neutral terms or with options labeled with relevant semantic content (for example, offer a corrupt deal). In addition, selfish monetary incentives in the bribery game were increased across within-subject conditions, whereas the payoffs for making no transaction or for playing the Trust Game remained fixed. Finally, to assess the moral connotation of decision-making scenarios, participants were asked to complete (post-decision) a Machiavellian scale, which has been previously associated with different aspects of moral decision-making. The experiment managed to identify a robust frame effect for both Players A and B. Results showed that both potential bribers and potential bribees were significantly less prone to choose the corrupt transaction in the loaded frame than in the abstract frame. In addition, the tendency for corruption increased as the selfish monetary incentives involved in the corrupt transaction increased across conditions. Moreover, responses to the game showed individual differences in the level of Machiavellianism, as high Machiavellian scores were directly associated with corrupt offers, though only in the loaded frame. The data also suggests that the present game managed to capture the expression of pro-social preferences (the idea of social preferences is that individuals evaluate an outcome not only on the basis of its consequences for themselves, but also based on consequences to others). This was evident in the fact that, in general, participants avoided the option that generated negative externalities when there was an option without externalities that was equivalent in terms of personal gain. In addition, possible ways of improving the sensitivity of the bribery game to variables expected to be associated with corruptibility were presented. These suggestions could be followed in future studies. The discussion draws attention to potential practical implications of present findings, which suggest that targeting socially undesired actions using labels with morally negative nuances could be an effective mean to discourage people from choosing them.

Keywords: Corruption; Framing; Experimental economics; Morality; Externalities.

\section{Introducción}

Los experimentos de coimas se presentan como herramientas complementarias para estudiar los determinantes psicológicos (comportamentales, cognitivos, emocionales) de la corrupción (Abbink, 2006; Bobkova y Egbert, 2012; Lambsdorff, 2012; Renner, 2004). Como evidencia explícitamente su definición estándar ("abuso del poder público confiado para obtener un beneficio privado", Aidt, 2003; Jain, 2001) la corrupción es un fenómeno que comporta una dimensión moral (Dungan, Waytz y Young, 2014; Hodgson 
y Jiang, 2007; Miller, 2010; Nichols y Robertson, 2017; Rose-Ackerman, 2006). Es más, todos los países tienen reglas (morales) más o menos explícitas que condenan la corrupción. En este sentido, es esperable que la corruptibilidad de las personas esté asociada a diferentes dimensiones morales (Haidt, 2001) $\mathrm{y}$, por ende, sería importante mostrar que los experimentos de corrupción logran captar esta dimensión psicológica.

Las implicancias morales de la corrupción pueden aparecer más o menos resaltadas en distintos aspectos de una situación experimental de coimas. Uno de los elementos clave lo constituyen las externalidades negativas (Abbink, Irlenbusch y Renner, 2002), es decir, el costo social de la corrupción, o en términos morales, el daño a terceros (Haidt, 2007). Un elemento indirecto es la existencia de sanciones que suelen indicar un comportamiento socialmente proscripto. En un juego de coimas enmarcado de manera neutra y de rondas repetidas con parejas estables, Abbink, Irlenbusch y Renner (2002) mostraron que los participantes eran sensibles a la posibilidad de recibir sanciones, pero no a producir externalidades negativas sobre los otros participantes de la sesión. La insensibilidad a grados crecientes de externalidades fue también el resultado obtenido por Barr y Serra (2009) en su versión neutra de un juego de coimas de una única ronda. De acuerdo con estos resultados, los juegos con marcos abstractos o neutros no parecerían contar con la dimensión moral antes mencionada, lo cual cuestiona su validez como instrumentos para estudiar la corrupción. A su vez, estos resultados sugieren que la mera estructura de pagos es insuficiente para representar una situación que en lo cotidiano implica la transgresión de normas.

Desde el punto de vista experimental, se ha recurrido a variaciones del marco para resaltar las connotaciones morales y normativas de una situación de coimas (Abbink y Hennig-Schmidt, 2006; Banerjee, 2016; Barr y Serra, 2009; Chaudhuri, Paichayontvijit, y Sbai, 2016). Las variaciones del marco comúnmente han involucrado cambios en los nombres asociados a los roles de los participantes (por ejemplo, "participante A" y "participante B" en la versión abstracta o neutra, versus "empresa" o "ciudadano" y "funcionario público" en la versión semánticamente cargada) y las palabras utilizadas para designar algunas de las acciones disponibles ("transferencia" versus "pago privado" o "coima" en los marcos neutro y cargado, respectivamente). No obstante, la comparación de estas variaciones del marco ha arrojado resultados mixtos: ausencia de efecto de marco (Abbink y Hennig-Schmidt, 2006; Cameron et al., 2009), presencia de efecto de marco (Banerjee, 2016; Chaudhuri, Paichayontvijit y Sbai, 2016); Barr y Serra (2009) presentan resultados mixtos dependientes del nivel de externalidad negativa involucrada.

En el experimento que se describe a continuación se explora el efecto de marco y las connotaciones morales en un juego de coimas. Con este fin, se tomaron algunas lecciones de la literatura de los juegos económicos de coimas, que se mencionan a continuación.

Primero, siguiendo la sugerencia de Barr y Serra (2009) de que la artificialidad de los roles (por ejemplo, empresa y funcionario) podría afectar la interpretación de las decisiones de los participantes como expresión de sus preferencias, no se utilizaron roles artificiales en los marcos del presente experimento. Tanto en el marco neutro como en el cargado, los participantes fueron denominados "jugador A", "jugador B" y "jugadores C". La diferencia entre marcos se limitó a los nombres de las acciones disponibles. Mientras que en el marco neutro las acciones se señalaron a través de letras, en el marco cargado las acciones llevaron rótulos con contenido semántico relevante (por ejemplo, “ofrecer un trato corrupto").

Segundo, las externalidades negativas en algunos juegos de coimas han sido representadas como consecuencias sobre los pagos de los demás participantes de la sesión (por ejemplo, Abbink, Irlenbusch y Renner, 2002; 
Abbink y Hennig-Schmidt, 2006). Esto introduce dos elementos que se confunden con la sensibilidad a producir daño: la posibilidad de reciprocidad negativa (decido corromperme porque pienso que los demás lo están haciendo, y me están perjudicando) y de competencia entre las parejas de la sesión (empresa y funcionario somos un equipo que compite contra las demás parejas). Por ello, en el procedimiento de este estudio, al igual que lo implementado por Barr y Serra (2009), la externalidad negativa de la corrupción la sufrieron terceros pasivos (los jugadores $\mathrm{C}$ ).

Tercero, un punto que podría ser relevante para determinar la capacidad del juego de coimas de detectar la sensibilidad a connotaciones morales tiene que ver con las opciones disponibles. Típicamente los juegos de coimas en la literatura de economía experimental presentan al jugador 1 solamente las opciones de ofrecer o de no ofrecer una coima (Abbink, Irlenbusch y Renner, 2002; Barr y Serra, 2009; Cameron et al., 2009). Por su parte, los jugadores 2 pueden aceptar o rechazar la coima (en el protocolo de Abbink, Irlenbusch y Renner (2002) también deciden si beneficiar o no al jugador 1; en los juegos de Barr y Serra (2009) y de Cameron et al. (2009) si el jugador acepta la coima automáticamente beneficia al jugador 1). Así, la única manera en que los participantes pueden cooperar es haciéndolo de manera corrupta, es decir, perjudicando a terceros. Esto enfrenta a los participantes al compromiso de entablar una relación cooperativa y perjudicar a terceros o no cooperar y no causar externalidades negativas. Dado que la cooperación puede ser una motivación preponderante y automática (Rand et al., 2012), el hecho de que la única opción cooperativa sea la corrupta podría estar disminuyendo la reacción moral de los participantes ante esta alternativa. Una solución sería ofrecer opciones de interacción más allá de la transacción corrupta. Incluso, sería ventajoso agregar opciones para minimizar un posible efecto de demanda del experimentador (Dawes, Fowler, Johnson, McElreath, y Smirnov, 2007; Zizzo, 2010). En especial, cuando las opciones disponibles son hacer (por ejemplo, ofrecer una coima) y no hacer nada (por ejemplo, no ofrecer la coima), podría haber un sesgo en los participantes por optar por la alternativa que implica una conducta activa (Pedersen, Kurzban y $\mathrm{McCu}$ 1logh, 2013). En base a estos razonamientos, en el juego de coimas presente se decidió incorporar una opción cooperativa que implicaba el riesgo típico de un dilema social, sin la posibilidad de perjudicar a terceros pasivos. Con la inclusión de esta opción los participantes podían optar por no realizar ninguna transacción, por una opción cooperativa no corrupta o por una opción cooperativa corrupta. Dependiendo del escenario, la opción corrupta presentaba incentivos materiales iguales o mayores (nunca menores) a la opción cooperativa no corrupta; esto permitió evaluar el nivel mínimo de incentivos materiales egoístas al que los participantes optaron por perjudicar a un tercero.

Por último, para evaluar la connotación moral de los escenarios de toma de decisiones evaluados, se solicitó a los participantes que completaran (post-decisión) una escala de maquiavelismo (Christie y Geis, 1970) con el objetivo de evaluar en qué medida variaciones en las respuestas al juego estaban asociadas a diferencias individuales en el nivel de maquiavelismo. El test de maquiavelismo se refiere al grado en que las personas son cínicas, emocionalmente desapegadas y manipuladoras (Bartels y Pizarro, 2011; Christie y Geis, 1970). El test mide ciertos atributos que lo hacen relevante para el trabajo presente. Por ejemplo, el maquiavelismo es un predictor confiable de comportamiento inmoral a lo largo de varias dimensiones, ya que se asocia a estándares éticos más bajos, una mayor intención de comportarse de manera inmoral en el futuro y menos escrúpulos en relación al comportamiento inmoral de otra personas (Jones y Paulhus, 2009). También el maquiavelismo está asociado a una visión más negativa y cínica del mundo y de los demás. Entre 
otras cosas, los maquiavélicos creen que las otras personas son más tramposas (Mudrack, 1993) o que tienen una disposición a engañar a los demás (Wirtz y Kum, 2004). Este test se ha usado también para predecir el comportamiento en experimentos económicos. Por ejemplo, Spitzer, Fischbacher, Herrnberger, Grön y Fehr (2007) encontraron que el maquiavelismo se asoció con transferencias más bajas en un juego de dictador, donde el monto de una transferencia se toma típicamente como indicativo de la preocupación por la equidad. En este sentido, se espera que los puntajes más elevados de Mach estén asociados a elecciones más frecuentes de la opción corrupta.

\section{Método}

\section{Participantes}

El experimento fue realizado en la Universidad Nacional del Sur (Bahía Blanca, Argentina), en el mes de noviembre de 2011. Los 126 participantes (77\% de mujeres) fueron reclutados asistiendo a un aula con previo consentimiento del profesor a cargo de la materia. Una vez en el aula, invitamos a los estudiantes presentes a participar en nuestro estudio de "toma de decisiones", resaltando explícitamente que la participación era voluntaria, y que podían ganar dinero tomando parte del estudio. El experimento se realizó en dos sesiones diferentes, tomando parte de la primera 72 estudiantes de la carrera de Ingeniería Química y en la segunda, 54 estudiantes de Bioquímica.

\section{Materiales y procedimiento}

Cada una de las dos sesiones siguió el orden siguiente: 1) un asistente introdujo el objetivo del experimento a los participantes ("estudiar la toma de decisiones"), explicó un escenario a modo de ejemplo, y mencionó asimismo cómo iban a determinarse los pagos al final de la sesión (más detalles abajo); 2) los cuader- nillos con los escenarios de decisión fueron distribuidos (i.e., se utilizó un procedimiento de papel-y-lápiz) (ver Apéndice I); y 3) una vez que todos los participantes devolvieron los cuadernillos a los asistentes, se realizaron los pagos correspondientes (i.e., se utilizaron incentivos monetarios reales).Asignación de roles y tratamientos: los cuadernillos diferían de acuerdo a si correspondían al rol $\mathrm{A}(\mathrm{n}=$ $53)$, al $B(n=53)$ o al $C(n=20)$ y al marco neutro $(n=53)$ o al cargado $(n=53)$, y fueron repartidos al azar entre los participantes.

Escenarios experimentales: Cada participante A o B respondió a 5 escenarios experimentales (1-5) y 2 escenarios controles (6-7). Todos los participantes A y B respondieron a los escenarios en el mismo orden (1-7) con el fin de hacer saliente el cambio creciente en los incentivos egoístas asociados a la opción corrupta en los escenarios 1-5. A través de los escenarios experimentales, se evaluó cómo respondieron los participantes al compromiso entre causar externalidades negativas y ganar más dinero. Los escenarios controles fueron utilizados para desambiguar la interpretación de ciertos patrones de elecciones en los escenarios experimentales. Los participantes $\mathrm{C}$ eran expuestos al juego, pero se les hacían preguntas en relación a cómo caracterizarían a un participante en función de sus decisiones (no se presentan el protocolo y los datos aquí por ser irrelevantes a los objetivos presentes).

Como se observa en el Cuadro 1, cada escenario experimental se compone de tres alternativas para los participantes $\mathrm{A}, \mathrm{y}$ dos alternativas en la rama izquierda y dos en la rama derecha del árbol de decisión para los participantes B. En el Cuadro 1 también se presentan los pagos asociados a cada combinación de decisiones para cada escenario. Los pagos asociados a las ramas $\mathrm{J} /$ confía $\mathrm{y} \mathrm{K} /$ sin intercambio no varían entre escenarios $\mathrm{y}$ además se caracterizan por no presentar externalidades negativas (los 10 participantes $\mathrm{C}$ ganan $\$ 100$ en total, independientemente de la decisión de B). En contraste, del escenario 1 al 5, se incrementaron sistemáticamente en 10 
$\%$ en cada escenario la ganancia que podían obtener los participantes si se coordinaban en la rama corrupta del árbol (L / Ofrece trato corrupto y P / Acepta; ver Cuadro 1). Si el trato corrupto se consumaba, es decir, si A elegía ofrecer el trato y B aceptaba, entonces los participantes $\mathrm{C}$ sufrían la externalidad negativa de la corrupción (ganaban \$0) y la ganancia de los participantes A y B se incrementaba (excepto en el escenario 1) respecto a la posibilidad de cooperación no corrupta (si A elegía $\mathrm{K}$ / confía y $\mathrm{B}$ elegía $\mathrm{N}$ / reciproca).

\section{Cuadro 1}

Pagos monetarios (en pesos argentinos) asociados a las distintas opciones de decisión para los participantes $A$ y $B$ en los escenarios 1-7.

\begin{tabular}{|c|c|c|c|c|c|c|}
\hline \multirow{3}{*}{\multicolumn{2}{|c|}{$\begin{array}{l}\text { opciones para A: } \\
\text { opciones para B: }\end{array}$}} & & \multicolumn{4}{|c|}{ OPCIONES } \\
\hline & & \multirow{2}{*}{\begin{tabular}{|l|}
$J /$ sin intercambio \\
\end{tabular}} & \multicolumn{2}{|c|}{$\mathrm{K} /$ confía } & \multicolumn{2}{|c|}{$L$ / ofrece trato corrupto } \\
\hline & & & \multirow[t]{2}{*}{ M / no reciproca } & \multirow[t]{2}{*}{$\mathrm{N} /$ reciproca } & \multirow[t]{2}{*}{ O / rechaza } & \multirow{2}{*}{ P/ acepta } \\
\hline escenario & participantes & & & & & \\
\hline \multirow[t]{3}{*}{$1 \mathrm{e}$} & A & 100 & 40 & 150 & 40 & 150 \\
\hline & B & 100 & 157 & 150 & 157 & 150 \\
\hline & Cs & 100 & 100 & 100 & 100 & 0 \\
\hline \multirow[t]{3}{*}{$2 e$} & A & 100 & 40 & 150 & 40 & 165 \\
\hline & B & 100 & 157 & 150 & 173 & 165 \\
\hline & Cs & 100 & 100 & 100 & 100 & 0 \\
\hline \multirow[t]{3}{*}{$3 e$} & A & 100 & 40 & 150 & 40 & 181 \\
\hline & B & 100 & 157 & 150 & 190 & 181 \\
\hline & Cs & 100 & 100 & 100 & 100 & 0 \\
\hline \multirow{3}{*}{$4 e$} & A & 100 & 40 & 150 & 40 & 199 \\
\hline & B & 100 & 157 & 150 & 209 & 199 \\
\hline & Cs & 100 & 100 & 100 & 100 & 0 \\
\hline \multirow[t]{3}{*}{$5 e$} & A & 100 & 40 & 150 & 40 & 219 \\
\hline & B & 100 & 157 & 150 & 230 & 219 \\
\hline & Cs & 100 & 100 & 100 & 100 & 0 \\
\hline \multirow[t]{3}{*}{$6 c$} & A & 100 & - & - & 110 & 150 \\
\hline & B & 100 & - & - & 150 & 157 \\
\hline & Cs & 100 & - & - & 100 & 0 \\
\hline \multirow[t]{3}{*}{$7 \mathrm{c}$} & A & 100 & - & - & 40 & 150 \\
\hline & B & 100 & - & - & 150 & 157 \\
\hline & Cs & 100 & & & 100 & 0 \\
\hline
\end{tabular}

Nota: En el tratamiento con marco neutro, las opciones de decisión eran nombradas por letras (J-P), mientras que en el marco cargado, llevaban nombres con contenido semántico relevante (por ejemplo, "ofrece un trato corrupto"). La letra junto al número de escenario indica si el escenario era experimental (e) o control (c).

En todos los escenarios experimentales, el participante B tenía incentivos egoístas para no reciprocar la confianza de $\mathrm{A}$ (rama izquierda) o no aceptar el trato corrupto (rama derecha). Es decir, que como ocurre en los dilemas sociales, la cooperación exitosa entre A y B (sea no corrupta o corrupta) dependía de, por un lado, la confianza de $\mathrm{A}$ en la reciprocidad de $\mathrm{B}$, y por otro lado, de que B, no traicione la confianza de A (Berg, Dickhaut y McCabe, 1995; Kollock, 1998).
Una vez que los participantes tomaban sus decisiones en los 5 escenarios experimentales, se implementaban los dos escenarios control (escenarios 6 y 7 en el Cuadro 1). Los escenarios control tenían como fin desambiguar la interpretación de algunos posibles comportamientos de los participantes A y B, a los fines de correlacionar sus decisiones con sus respuestas a la escala de Maquiavelismo Mach IV (Christie y Geis, 1970). Una posible fuente de ambigüedad consistía en que un participante 
A eligiera siempre la opción $\mathrm{J} /$ sin intercambio a lo largo de los 5 escenarios experimentales. En tal caso, no podría saberse si ese participante estaba motivado por evitar externalidades negativas (una conducta pro-social) o por aversión al riesgo y evitar la posibilidad de obtener solo la ganancia mínima (\$40). El escenario 6 permite desambiguar esa situación, pues cuenta con 2 opciones para los participantes A: una opción sin externalidades en la que, sin intercambio, A y B ganan $\$ 100$, y los C también, y una opción corrupta (con externalidades) pero sin riesgo, en la que, si B acepta el trato corrupto, A gana $\$ 150$, B gana $\$ 157$ y los C $\$ 0$, mientras que si B rechaza el trato corrupto, A gana $\$ 110$ (más que en la opción sin intercambio), B gana $\$ 150$ (menos que en la opción corrupta) y los $\mathrm{C}$ ganan $\$ 100$. Es decir, que las elecciones de un participante A que opta por $\mathrm{J} / \mathrm{sin}$ intercambio a lo largo de los 5 escenarios experimentales solo pueden considerarse como motivadas moralmente (evitar el daño a terceros) si en el escenario control 6 también elige la opción de no ofrecer el trato corrupto a pesar de que implica un costo egoísta.

Una posibilidad ambigua respecto a las decisiones de $\mathrm{B}$ es que siempre elija rechazar la oferta corrupta de A en los escenarios 1-5. Tal serie de decisiones podría estar dirigida a evitar externalidades negativas y castigar la decisión corrupta de $\mathrm{A}$ u obtener la mayor ganancia egoísta. Así, el escenario 7 comienza con A contando con dos opciones, una sin intercambio en la que todos terminan con $\$ 100$, y una con oferta de trato corrupto. A diferencia del escenario 1, en donde aceptar el trato corrupto implica sacrificar $\$ 7$ para B (pues gana $\$ 150$ si lo acepta y $\$ 157$ si lo rechaza; ver Cuadro 1), en el escenario 7, aceptar el trato corrupto, además de producir externalidades, implica ganar \$157 en lugar de \$150. Es decir, que las elecciones de un participante $\mathrm{B}$ que opta por $\mathrm{O} /$ rechazar [la oferta corrupta] a lo largo de los 5 escenarios experimentales solo pueden considerarse como motivadas moralmente (evitar el daño a terceros y castigar la elección corrupta de A) si en el escenario control 7 también elige la opción de rechazar el trato corrupto a pesar de que implica un costo egoísta.

Por último, los participantes completaron una versión en español de la escala de Maquiavelismo Mach IV (Christie y Geis, 1970), que se compone de 20 enunciados, tales como "Uno debe actuar solo cuando hacerlo es moralmente correcto" o "Quien confía completamente en cualquiera, busca problemas". Los participantes deben expresar su nivel de acuerdo con cada enunciado en una escala de Likert de 5 puntos que va de "totalmente de acuerdo" a "en total desacuerdo". Luego, completaron un cuestionario socio-demográfico y entregaron los cuadernillos. En cada sesión, recibieron un pago en efectivo 12 participantes: un participante A y un participante B elegidos al azar, y los 10 participantes C. Los participantes A y $\mathrm{B}$ recibieron sus pagos de acuerdo a las decisiones que tomaron en uno de los 5 escenarios elegidos al azar. En el caso de los participantes A y B, se le pidió al participante A (B) seleccionado que eligiera al azar un cuadernillo de un participante B (A) (cuya identidad no era revelada) de entre todos los cuadernillos de los participantes B (A) de esa sesión. El pago de ese A (B) se determinó de acuerdo a las elecciones que realizaron ambos en el escenario seleccionado. En el caso de los 10 participantes C, cada uno debía seleccionar al azar dos cuadernillos, uno correspondiente a un participante $\mathrm{A}$ y otro a un participante B (cuyas identidades no eran reveladas), y sus pagos se determinaban a partir de las elecciones que habían realizado dichos participantes en el escenario seleccionado. El método de compensación ha sido utilizado previamente en otros estudios experimentales sobre corrupción (por ejemplo, Schulze y Frank, 2003).

\section{Variables dependientes}

\section{Participantes $A$}

Se definió la variable frecuencia de oferta [corrupta] sumando la cantidad de veces que el participante eligió la opción L / ofrece un trato 
corrupto a lo largo de los 7 escenarios. Además, para capturar el efecto del incremento del incentivo monetario egoísta en la rama con posibilidad de externalidades negativas, se definió una variable que llamamos mínimo incentivo de corrupción (MIC) que indica el primer escenario experimental en el que el participante $\mathrm{A}$ escogió la opción L / ofrece un trato corrupto. La variable MIC tiene un rango de 1 a 6 , donde 1 significa que el participante escogió la opción corrupta ya en el escenario 1 (i.e., sin incentivo egoísta), 5 que la eligió recién en el escenario 5 (i.e., solo con el incentivo más sustancioso), y 6 que el participante nunca eligió la opción corrupta. Si un participante eligió ofrecer el trato corrupto en el escenario control 6, su variable MIC adquiere valor de 1 (i.e., decide corromperse con un bajo incentivo).

\section{Participantes $B$}

Utilizando el método de estrategias (Selten, 1967), se registraron las decisiones de B para cada una de las posibles decisiones de A, es decir, dos decisiones por cada escenario experimental ( $\mathrm{M} /$ no recíproca y $\mathrm{N}$ / recíproca en la rama $\mathrm{K}$ / confía, y $\mathrm{O}$ / rechaza y $\mathrm{P}$ / acepta en la rama $\mathrm{L} /$ ofrece un trato corrupto). Se calculó la variable frecuencia de corrupción para cada participante B sumando la cantidad de veces que $\mathrm{B}$ optó por $\mathrm{P}$ o aceptó [un trato corrupto] en los 7 escenarios. Asimismo, también se calculó la variable MIC para los jugadores B, que indica el primer escenario experimental en el que el participante $\mathrm{B}$ escogió $\mathrm{P}$ o aceptó el trato corrupto. La variable MIC para B tiene el mismo rango y sentido que lo arriba descripto para el participante A. Si un participante eligió aceptar el trato corrupto en el escenario control 7, su variable MIC adquiere valor de 1 (i.e., con un bajo incentivo decide corromperse).

\section{Análisis estadísticos}

Los análisis estadísticos se realizaron y se presentan de manera independiente para los jugadores A y para los B.
En primera instancia, se utilizó estadística no paramétrica dado que las variables que se estudiaron no presentaron distribuciones normales (test de normalidad de Shapiro-Wilk: frecuencia de oferta (solo participantes A), $w=.87, p<.05$; frecuencia de corrupción (solo participantes $\mathrm{B}$ ), $w=.83, p$ $<.05$; MIC para los participantes $\mathrm{A}, w=.76$, $p<.05$; MIC para los participantes $\mathrm{B}, w=$ $.71, p<.05)$. El test $U$ de Mann-Whitney se usó para comparar la frecuencia de ofertas, la frecuencia de ocorrupción y el MIC entre tratamientos (marco neutro versus cargado). Por último, se utilizó la prueba de correlaciones por rangos de Spearman para evaluar la asociación entre los valores de la escala Mach IV y las variables frecuencia de ofertas, frecuencia de corrupción y las MIC.

En segundo lugar, se recurrió a modelos de regresión logística multinivel debido al diseño con medidas repetidas. Los modelos multinivel toman en consideración y permiten dar cuenta de la estructura jerárquica de los datos (Snijders y Bosker, 1999), así como manejar datos en los que las observaciones no son independientes entre sí. De los 126 participantes, 106 respondieron a los 5 escenarios experimentales más los 2 escenarios de control (en cada sesión 10 participantes fueron pasivos), por lo que se tienen 742 observaciones (que corresponden al Nivel 1) anidadas en 107 sujetos (Nivel 2). Las condiciones o tratamientos experimentales son variables de Nivel 2. Como método de estimación de los coeficientes usamos Cadenas de Markov de Monte Carlo (MCMC), ya que este procedimiento arroja estimaciones menos sesgadas que los métodos de estimación aproximada estándar. Además, MCMC calcula el desvío estadístico, lo que permite utilizar un test formal de Chi cuadrado para comparar modelos estadísticos anidados (Hox, 1995). Valores significativos indican que el modelo con el desvío menor es una mejora con respecto al modelo con mayor desvío. Los modelos se estimaron utilizando MLwiN a través del comando runmlwin de Stata (ver Leckie y Charlton, 2012). 


\section{Resultados}

\section{Participantes A}

La Figura 1 muestra el porcentaje de participantes A que eligieron ofrecer el trato corrupto en función del marco y el escenario experimental. El promedio de la variable "frecuencia de ofertas" fue de más del doble en el marco neutro que en el marco cargado (media \pm 1 EEM: $3.19 \pm .34$ en el marco neutro, y $1.41 \pm .25$ en el marco cargado; Mann-Whitney $U$ test, $U_{26,27}=93, \mathrm{z}=4.59, p$ $<.001)$.

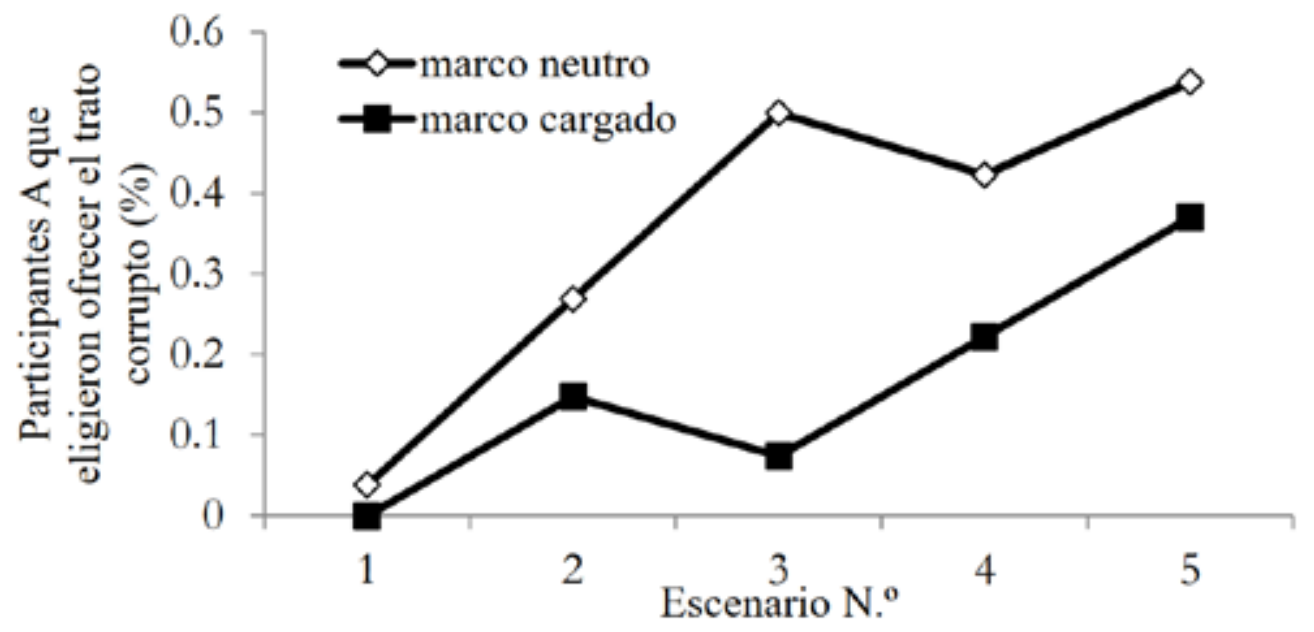

Figura 1. Porcentaje de participantes A que eligieron la opción $L$ en el marco abstracto $(n=26)$ o que eligieron "ofrece trato corrupto" en el marco cargado $(n=27)$.

Nota: El eje horizontal representa los cinco escenarios de decisión. El incentivo monetario asociado a la opción L y a la opción "ofrece trato corrupto" se aumentó $10 \%$ en cada escenario, mientras que los pagos en las opciones alternativas permanecieron fijos.

Fuente: elaboración propia.

Respecto a la variable MIC, se encontró que en promedio, los participantes A eligieron la opción con posibilidad de externalidades negativas con menores incentivos egoístas en el marco neutro que en el marco cargado (media \pm 1 EEM: $1.46 \pm .27$ en el marco neutro, y 3.07 \pm .44 en el marco cargado; Mann-Whitney $U$ test, $\left.U_{26,27}=199, z=-2.70, p<.01\right)$.

Por último, se evaluó la correlación entre los puntajes de maquiavelismo y las variables "frecuencia de oferta" y MIC. Es importante remarcar que los puntajes de maquiavelismo no difirieron significativamente entre marcos (Mann-Whitney $U$ test, $U_{27,26}=347,5, z=$ $-.06, p=.95)$. En el marco neutro, la asociación entre los puntajes de maquiavelismo y la frecuencia de oferta o la MIC no fue significativa $(\rho=.19, n=27, p>.05 ; \rho=-.25, n=27$, $p>.05$, respectivamente). En contraste, en el marco cargado, la asociación de los puntajes de maquiavelismo fue significativa tanto con la frecuencia de ofertas como con la MIC ( $\rho=$ $.46, \mathrm{n}=26, p<.05 ; \rho=-.45, \mathrm{n}=26, p<.05$, respectivamente).

Los participantes A poseían tres opciones: sin intercambio (SI), confía (C) y ofrece trato corrupto (TC). La Tabla 1 reporta los resultados de una regresión multinomial con intercepto aleatorio. La variable dependiente es Acción y toma uno de tres valores $(\mathrm{SI}=1, \mathrm{C}$ $=2$ y TC $=3$ ). La variable Escenario_centrado se definió tomando el promedio de las rondas, por lo tanto Escenario_centrado = Escenario - 4. Por consiguiente los valores de Escenario_centrado están en un rango que va de -3 a 3 . También se centró la variable Mach. Tratamiento es una variable dicotómica que toma valor 0 para el tratamiento neutro y valor 1 para el marco cargado. Como base de contraste se utilizó $\mathrm{TC}=3$. 
Tabla 1

Regresión logística multinomial (Markov Chain Monte Carlo estimation). Variable dependiente: Acción $(S I=1 ; C=2 ; T C=3)$.

\begin{tabular}{|c|c|c|c|c|c|}
\hline SI (1 vs 3 ) & \multirow[b]{2}{*}{ Intercepto 1} & Coeficiente & D. E. & \multicolumn{2}{|c|}{ [Intervalo de confianza] } \\
\hline & & -.084 & .233 & -.560 & .363 \\
\hline & Escenario_cen & $-.290 * * *$ & .0766 & -.437 & -.135 \\
\hline & Tratamiento & $1.355 * * *$ & .361 & .665 & 2.097 \\
\hline & Mach_cen & $-.058 * * *$ & .020 & -.102 & -.020 \\
\hline \multicolumn{6}{|c|}{ Confia (2 vs 3 ) } \\
\hline & Intercepto2 & $-1.867 * * *$ & .457 & -2.818 & -1.004 \\
\hline & Escenario_cen & $-.870 * * *$ & .118 & -1.115 & -.647 \\
\hline & Tratamiento & $2.155^{* * *}$ & .560 & 1.119 & 3.285 \\
\hline & Mach_cen & $-.0512 *$ & .031 & -.121 & .011 \\
\hline \multicolumn{6}{|c|}{ Parte Aleatoria } \\
\hline & $\operatorname{Var}($ int1) & .565 & .358 & .117 & 1.455 \\
\hline & $\operatorname{Cov}($ in 1, int 2$)$ & .375 & .520 & -.416 & 1.596 \\
\hline & $\operatorname{Var}($ int2) & 2.012 & 1.205 & .0380 & 4.810 \\
\hline
\end{tabular}

Observaciones

371

$* * * p<.001 ; * * p<.05 ; * p<.10$.

Nótese que se tomó como base de contraste $\mathrm{TC}=3$. La variable esc_centrado es significativa en los dos contrastes $(p<.001)$, y tiene el signo negativo esperado, lo que indica que, respecto de $\mathrm{TC}$, a medida que aumentan las rondas disminuye la probabilidad de elecciones de SI y C. El patrón opuesto se observa en la variable tratamiento, también significativa en los dos contrastes, y con el signo positivo esperado, lo que indica que en el tratamiento cargado aumenta de manera significativa la probabilidad de que los participantes elijan tanto SI como C frente a TC. En el caso de la variable Mach, es significativa con el signo esperado (negativo) en ambos contrastes (contraste $1, p<.001$; contraste $2, p$ $<.10$ ), lo que indica que un puntaje mayor en la escala acrecienta la probabilidad de elegir TC.

\section{Participantes B}

La Figura 2 muestra el porcentaje de participantes B que eligió la opción con externalidades negativas. La frecuencia de corrupción fue superior en el marco neutro que en el cargo (media \pm 1 EEM: $1.85 \pm .36$ en el marco neutro, y $1.65 \pm .40$ en el marco cargado), aunque no de manera significativa (Mann-Whitney $U$ test, $U_{27,26}=323, z=.50, p$ $=.62$; ver modelo 2 de la Tabla 2). Cuando se introduce la variable tratamiento, la diferencia en desvío respecto del modelo 1 es solo .701, que no resulta significativo $(p=.31)$. 


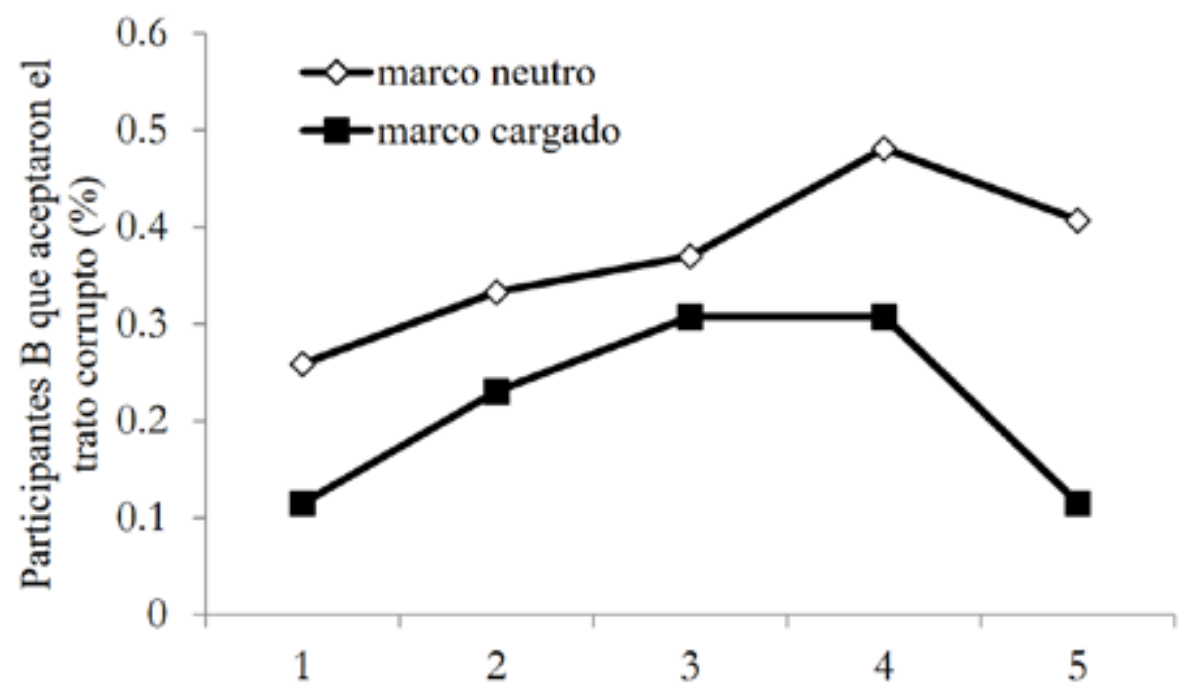

Escenario $\mathrm{N}^{\circ}$

Figura 2. Porcentaje de participantes B que aceptaron el trato corrupto graficado en función de los escenarios y los tratamientos.

Respecto a la variable MIC, los participantes $\mathrm{B}$ en el marco neutro eligieron la opción corrupta con un incentivo egoísta menor que en el marco cargado (media \pm 1 EEM: 2.44 \pm .42 en el marco neutro, y $3.69 \pm .45$ en el marco cargado; Mann-Whitney $U$ test, $U_{27,26}$ $=231.5, Z=-2.13, p<.05)$. Este resultado también se ve confirmado en la regresión ordinal que reportamos en la Tabla 3. Nótese que el coeficiente para la variable tratamiento resulta significativo en la dirección esperada $(p<.05$; ver Tabla 3$)$. Para la variable tratamiento entonces, se dice que para un incremento de la variable (esto es, de 0 a 1 ), se espera un aumento de 3.05 en la probabilidad de estar en la categoría anterior (se toma el valor más alto de MIC como base de comparación)

Tabla 2

Regresión logística multinivel (Markov Chain Monte Carlo estimation). Variable dependiente: Acción (0 = rechazar; 1 = aceptar).

\begin{tabular}{c|c|c|c|c}
\multicolumn{2}{c}{ Modelo nulo } & Modelo 1 & Modelo 2 & Modelo 3 \\
\hline & Coef $(\mathrm{ES})$ & Coef $(\mathrm{ES})$ & Coef $(\mathrm{ES})$ & Coef $(\mathrm{ES})$ \\
Intercepto & $-1.240(.357)$ & $-1.283(.380)$ & $-.578(.566)$ & -.747 \\
Esc_centrado & & $.232(.076)^{* * *}$ & $.239(.078)^{* * *}$ & $.244(.076)^{* * *}$ \\
Tratamiento & & & $-1.504(.808)$ & $-1.384(.774)$ \\
Mach & & & & $.080(.053)$ \\
Parte aleatoria & & & & \\
\hline Varianza entre sujetos & $5.138(1.930)$ & $5.868(2.367)$ & $5.615(2.223)$ & $5.927(2.269)$ \\
Desvío & 306.095 & 294.586 & 293.885 & 291.965 \\
Dif. en desvío & & 11.49 & .701 & 1.92 \\
\hline Observaciones & 371 & & & \\
$* * * p<.001$. & & &
\end{tabular}


Por último, se analizó la correlación entre los puntajes de maquiavelismo de los participantes B y la frecuencia de corrupción y la MIC. Los puntajes de maquiavelismo no difirieron significativamente entre marcos (Mann-Whitney $U$ test, $U_{27,26}=261, Z=1.60$, $p>$.10) y tampoco se encontró evidencia de que exista una asociación significativa entre los puntajes de maquiavelismo y las decisiones de los participantes B (marco neutro: asociación entre la frecuencia de corrupción $\mathrm{y}$ el puntaje de maquiavelismo, $\rho=-.07, n=$
27, $p>$.05; asociación entre MIC y el puntaje de maquiavelismo, $\rho=.03, n=27, p>.05$; marco cargado: asociación entre la frecuencia de corrupción y el puntaje de maquiavelismo, $\rho=.25, n=26, p>.05$; asociación entre MIC y el puntaje de maquiavelismo $\rho=-.24, n=$ $27, p>.05)$. El coeficiente para maquiavelismo no es significativo en la regresión logística (introducir la variable Mach no mejora el ajuste del modelo, diferencia en desvío: 1.92, $p=.16$; ver Tabla 2 , modelo 3 ) y tampoco lo es en la regresión logística ordinal $(p>.10)$.

Tabla 3

Regresión logística ordinal. Variable dependiente: MIC.

\begin{tabular}{c|c|c|c|c} 
& $\beta$ & E.E & $\mathrm{z}$ & $\begin{array}{c}{[95 \% \text { intervalos de }} \\
\text { confianza }]\end{array}$ \\
\cline { 2 - 5 } Sexo & .863 & .539 & -.24 & {$[.25,2.93]$} \\
Tratamiento & $3.07 * *$ & 1.71 & 2.02 & {$[1.03,9.15]$} \\
Mach & .954 & .037 & -1.20 & {$[.880,1.02]$} \\
\hline Observaciones & 53 & &
\end{tabular}

$* * \mathrm{p}<.05$.

\section{Discusión y conclusión}

El objetivo principal del presente estudio consistió en evaluar si un juego económico de coimas logra reflejar de manera adecuada la corruptibilidad de los participantes, entendida como una dimensión moral de la corrupción. Se argumenta que la transgresión moral es un elemento definitorio de la corrupción (ver referencias en la introducción) y por lo tanto es crucial saber si las respuestas de los participantes reflejan dicha dimensión moral. En el experimento, los participantes A y B podían elegir acciones que reflejaban motivaciones de confianza y reciprocidad, o acciones que podían generar externalidades negativas sobre terceros pasivos. Se obtuvieron decisiones en dos grupos independientes, ya sea que participaran en un juego enmarcado de forma neutra o que lo hicieran en un juego cuyas opciones llevaban nombres con contenido semántico relevante.

A diferencia de Abbink y Hennig-Schmidt
(2006) y Barr y Serra (2009) que no encontraron efectos robustos de marco a través de los roles, el experimento aquí descripto logró identificar un claro efecto de marco tanto para los jugadores A como para los B. Una razón del éxito del presente experimento en este punto particular podría consistir en que las variaciones en el marco evitaron referencias a roles hipotéticos tales como el de Firma o Funcionario Público. Algunos autores sostienen que cuando los roles experimentales resultan extraños, pueden parecer demasiado artificiales a los participantes e inducirlos a adoptar un comportamiento similar al que adoptarían en un juego de roles en lugar de expresar sus preferencias verdaderas (Bardsley, 2005; Barr y Serra, 2009). En cambio, solo etiquetando las acciones en el marco cargado se logró un efecto potente sobre las decisiones de los participantes, llevándolos a evitar en mayor medida las alternativas connotadas de manera negativa que cuando las acciones eran nombradas de manera abstracta. 
Los datos sugieren asimismo que el presente juego logró capturar la expresión de preferencias pro-sociales (la idea de preferencias sociales consiste en que los individuos evalúan un resultado no solo sobre la base de las consecuencias que tiene para ellos mismos, sino en función también de las consecuencias hacia otros; por ejemplo, Bolton y Ockenfels, 2000; Fehr y Schmidt, 1999). Esto se hizo patente en el hecho de que, en general, los participantes evitaron la opción que generaba externalidades negativas cuando había una opción sin externalidades que era equivalente en términos de ganancias personales. Se encontró este efecto para los participantes A, es decir, los potenciales oferentes de coimas, en ambos marcos. Para los participantes B, el efecto solo estuvo presente en el marco cargado. Esta asimetría entre roles podría deberse a que los participantes B concibieran la rama corrupta del árbol en el marco neutro como una decisión inevitable entre perjudicar a los participantes A o a los C. En este sentido, puede ser que hayan percibido las opciones como equivalentes en términos morales, lo que explicaría el motivo por el cual no exhibieron una preferencia en contra de la opción con externalidades.

El acuerdo generalizado es que la corrupción tiene efectos negativos sobre el bienestar de una sociedad (Ambraseys y Billam, 2011, Mauro, 1995; Méon y Sekkat, 2005; Rose-Ackerman, 2006). Sin embargo, varios autores conciben la posibilidad de que, en ciertos casos, la corrupción incremente la eficiencia de las transacciones (Bardhan, 1997; Huntington, 1968; Leff, 1964; Leys, 1965). Esto se relaciona con una característica de los parámetros empleados en el juego presente. Nótese que a partir del segundo escenario, la suma de pagos es mayor en la rama L/Ofrecer trato corrupto que en las demás alternativas del juego. Esto significa que los participantes A pueden haber estado motivados por razones de eficiencia para elegir la rama corrupta del juego. No obstante, los resultados del efecto de marco se muestran robustos ante este aspecto del diseño presente ya que se observa que el marco cargado disuadió a los participantes de elegir la rama corrupta del juego a pesar de los incrementos de eficiencia asociados a dichas opciones.

Con el objetivo de capturar diferencias individuales en una dimensión moral relevante, se implementó el cuestionario de maquiavelismo. En estudios previos, se ha mostrado que las personas con puntajes altos en maquiavelismo tienden a exhibir la característica actitud del Homo economicus, es decir, de búsqueda de la maximización de los pagos egoístas, independientemente del efecto de las acciones sobre el pago de los otros (Christie y Geis, 1970; Gunnthorsdottir, McCab y Smith, 2002; Spitzer et al., 2007). De manera consonante, el maquiavelismo está asociado negativamente con adhesión a preceptos deontológicos (Bartels y Pizarro, 2011). Por lo tanto, se esperaba que los participantes con puntajes más altos en maquiavelismo estuvieran más inclinados a elegir la opción con externalidades negativas en el juego, ya que presumiblemente tendrían menor sensibilidad a los costos psicológicos o morales implicados en la corrupción. En efecto, en el marco cargado se encontraron correlaciones significativas (con los signos esperados) entre el puntaje de maquiavelismo de los potenciales oferentes de coimas y las variables que se construyeron para medir la corruptibilidad de los sujetos. Sin embargo, no se encontró ninguna asociación entre los puntajes de maquiavelismo y las decisiones de los potenciales oferentes de coimas en el marco neutro ni con las decisiones de los participantes $\mathrm{B}$ en ninguno de los dos marcos. Estos resultados negativos parecen sugerir que la dimensión moral capturada por la prueba Mach IV puede no coincidir con la medida por el presente juego. También puede ser el caso que el presente experimento no haya resultado ser lo suficientemente potente para detectar una asociación entre maquiavelismo y corruptibilidad en todos los casos. Esto podría ser así debido al menos a tres razones. En primer lugar, la dispersión de 
puntajes Mach en nuestro experimento resultó ser relativamente baja, especialmente si la comparamos con otros estudios en los que los autores categorizan a los sujetos en low-Mach (los que puntúan dos desvíos estándar por debajo de la media) y high-Mach (los que puntúan dos desvíos estándar por encima de la media), es decir que solo toman en cuenta los casos extremos (Gunnthorsdottir et al., 2002). Desde luego ello podría corroborarse implementando el presente diseño con una muestra lo suficientemente grande. En segundo lugar, la externalidad negativa permanecía fija a lo largo de los escenarios, lo que puede haber limitado la expresión de una preferencia por no dañar a terceros. Este problema podría ser subsanado implementando una variante del juego en la que las externalidades también aumenten o disminuyan dependiendo de la condición experimental. Y en tercer lugar, las alternativas de decisión para B no incluían la posibilidad de que los B pudieran rechazar la coima $\sin$ al mismo tiempo maximizar sus ganancias. Esto puede resultar problemático porque, de esta manera, rechazar el trato corrupto pudo resultar moralmente ambiguo para los participantes, en especial en el marco neutro (el escenario 7 controlaba parcialmente esa opción). Como se ha visto en otros contextos, la existencia de incentivos externos puede limitar o incluso ir en contra de la expresión de comportamientos pro-sociales intrínsecamente motivados (Bowles y Poliana-Reyes, 2012; Schulze y Frank, 2003).

En conclusión, las diferencias del marco sobre las decisiones sugieren que, al definir las acciones del juego con contenido semántico relevante, aumentarían los costos morales de producir externalidades negativas. Esto llevaría a los participantes a evitar las acciones corruptas en mayor medida que cuando el marco no explicita las normas en juego. Esto se ve corroborado para los participantes A (posibles oferentes de tratos corruptos) en función de sus respuestas a la escala de maquiavelismo. Los participantes que reportaron menor disposición a considerar los costos sociales de sus acciones efectivamente reflejaron esa actitud en sus decisiones en el juego con el marco cargado.

Para finalizar, estos resultados conducen a considerar una implicación potencial del estudio presente como principio conductual preliminar a considerar en el diseño de políticas públicas que busquen combatir la corrupción. Es evidente que diferentes marcos pueden provocar diferentes respuestas en los participantes y, por lo tanto, es de sumo interés determinar qué marcos podrían tener mayor eficacia a la hora de implementar políticas públicas tendientes a combatir la corrupción. Los resultados presentes sugieren que nombrar aquellas acciones socialmente no deseadas con etiquetas que señalen matices moralmente negativos podría ser un medio eficaz para disuadir a las personas de seguir dichas acciones.

Ahora bien, las etiquetas que se usen pueden ser muy diversas y tener resultados dispares. Aquí puede orientarnos la literatura de comunicación sobre marco. Sabido es que la discusión pública en torno a la corrupción está fuertemente signada por los marcos éticos de referencia que los medios de comunicación y que las elites políticas avanzan. La literatura sobre value-framing concibe el fenómeno de la siguiente manera: "concebimos el encuadre en términos de los diferentes conjuntos de valores que proporcionan la justificación subyacente para discusiones políticas particulares" (Shah, Domke y Wackman, 1996). Los autores continúan interpretando el concepto de la siguiente manera: "el encuadre ético de los asuntos políticos activa consideraciones sobre los derechos y la moral en la mente de algunos ciudadanos." (Shah, Domke y Wackman, 2001, p. 229).

En general, hay dos tipos de valores que suelen enmarcar las discusiones públicas: de un lado, valores éticos, que suelen estar vinculados al discurso en torno a los derechos y los principios básicos; de otro, valores materiales, que se vinculan a discusiones económicas. Sin duda la corrupción es un tema que involucra 
ambas dimensiones valorativas. Por ejemplo, sería interesante investigar diferentes marcos que hagan más salientes aspectos económicos como la ineficiencia, o aspectos morales como el daño a terceros, ya que pueden tener efectos diferentes en los participantes. El resultado encontrado en este trabajo y las líneas de investigación que sugiere no solo podrían ser beneficiosas (o útiles) para las campañas anti-corrupción, sino también para aplicar en otras circunstancias en las que se desee lograr un mayor cumplimiento de las normas.

\section{Referencias bibliográficas}

Abbink, K. (2006). Laboratory experiments on corruption. En S. Rose-Ackerman (Ed.), The Handbook of Corruption (p. 418-437). UK y Northampton, USA: Edward Elgar Publishers, Cheltenham.

Abbink, K. y Hennig Schmidt, H. (2006). Neutral versus Loaded Instructions in a Bribery Experiment. Experimental Economics, 9(2), 103-121. https://doi.org/10.1007/s10683-006-5385-z

Abbink, K., Irlenbusch, B. y Renner, E. (2002). An Experimental Bribery Game. Journal of Law, Economics, and Organization, 18(2), 428-454. https://doi.org/10.1093/jleo/18.2.428

Aidt, T. (2003). Economic analysis of corruption: a survey. The Economic Journal, 113(491), 632-652. $\quad$ https://doi.org/10.1046/j.00130133.2003.00171.x

Ambraseys, N., y Billham, R. (2011). Corruption kills, Nature 469, 153-155. https://doi.org/10.1038/469153a

Banerjee, R. (2016). On the interpretation of bribery in a laboratory corruption game: moral frames and social norms. Experimental Economics, 19(1), 240-267. https://doi.org/10.1007/ s10683-015-9436-1

Bardhan. P. (1997). Corruption and development: A review of issues. Journal of Economic Literature, 35(5), 1320-1346.

Bardsley, N. (2005). Experimental Economics and the Artificiality of Alteration. Journal of Economic Methodology, 12(2), 239-251. https://doi.org/10.1080/13501780500086115
Barr, A. y Serra, D. (2009). The effects of externalities and framing on bribery in a petty corruption experiment. Experimental Economics, 12(4), 488-503. https://doi.org/10.1007/ s10683-009-9225-9

Bartels, D. y Pizarro, D. (2011). The mismeasure of morals: Antisocial personality traits predict utilitarian responses to moral dilemmas. Cognition, 121(1), 154-161. https://doi. org/10.1016/j.cognition.2011.05.010

Berg, J., Dickhaut, J. y McCabe, K. (1995). Trust, reciprocity and social history. Games and Economic Behavior, 10(1), 122-142. https:// doi.org/10.1006/game.1995.1027

Bobkova, N. y Egbert, H. (2012). Corruption investigated in the lab: A Survey of the Experimental Literature. International Journal of Latest Trends in Finance and Economic Sciences, 2(4), 337-349.

Bolton, G. y Ockenfels, A. (2000). ERC: A Theory of Equity, Reciprocity, and Competition. American Economic Review, 90(1), 166-193. https://doi.org/10.1257/aer.90.1.166

Bowles, S. y Polanía-Reyes, S. (2012). Economic incentives and social preferences: substitutes or complements? Journal of Economic Literature, 50(2), 368-425. https://doi.org/10.1257/ jel.50.2.368

Cameron, L., Chaudhuri, A., Nisvan, E. y Gangadharan, L. (2009). Propensities to Engage in and Punish Corrupt Behavior: Experimental Evidence from Australia, India, Indonesia and Singapore. Journal of Public Economics, 93(7/8), 843-851. https://doi.org/10.1016/j. jpubeco.2009.03.004

Chaudhuri, A., Paichayontvijit, T. y Sbai, E. (2016). The Role of Framing, Inequity and History in a Corruption Game: Some Experimental Evidence. Games 7(2), 13. https://doi. org/10.3390/g7020013

Christie, R., y Geis, F.L. (1970). Studies in Machiavellianism, New York: Academic Press.

Dawes, C. T., Fowler, J. H., Johnson, T., McElreath, R. y Smirnov, O. (2007). Egalitarian motives in humans. Nature 446(7137), 794-796. https://doi.org/10.1038/nature05651

Dungan, J., Waytz, A. y Young, L. (2014). Corrup- 
tion in the context of moral trade-offs. Journal of Interdisciplinary Economics, 26(1-2), 97-118. https://doi.org/10.1177/0260107914540832

Fehr, E. y Schmidt, K. (1999). A Theory of Fairness, Competition, and Cooperation. Quarterly Journal of Economics, 114(3), 817-868. https:// doi.org/10.1162/003355399556151

Gunnthorsdottir, A., McCabe, K. y Smith, V. (2002). Using the Machiavellianism instrument to predict trustworthiness in a bargaining game. Journal of Economic Psychology, 23(1), 49-66. https://doi.org/10.1016/s01674870(01)00067-8

Haidt, J. (2001). The emotional dog and its rational tail: a social intuitionist approach to moral judgment. Psychological Review, 108(4), 814-834.

https://doi.org/10.1037/0033-295x.108.4.814

Haidt, J. (2007). The new synthesis in moral psychology, Science, 316(5827), 998-1002. https://doi.org/10.1126/science.1137651

Hodgson, G.M. y Jiang, S. (2007). The economics of corruption and the corruption of economics: An institutionalist perspective. Journal of Economic Issues, 41(4), 1043-1061. https:// doi.org/10.1080/00213624.2007.11507086

Hox, J. J. (1995). Applied Multilevel Analysis, Amsterdam: TT-Publikaties.

Huntington, S. (1968). Political Order in Changing Societies. New Haven: Yale University Press.

Jain, A. K. (2001). Corruption: A review. Journal of Economic Surveys, 15(1), 71-121. https:// doi.org/10.1111/1467-6419.00133

Jones, D.N., y Paulhus, D.L. (2009). Machiave1lianism. En M.R. Leary y R.H. Hoyle (Eds.), Handbook of individual differences in social behavior (p. 257-273). New York/London: The Guilford Press.

Kollock, P. (1998). Social Dilemmas: The Anatomy of Cooperation. Annual Review of Sociology, 24(1), 183-214. https://doi. org/10.1146/annurev.soc.24.1.183

Lambsdorff, J.G. (2012). Behavioral and experimental economics as a guidance to anticorruption. En Serra, D.y Wantchekon,L.(Eds.)(2012) New advances in the experimental research on corruption (Research in Experimental Econo- mics, Volume 15) (p. 279-300) .UK: Emerald Group Publishing Ltd. https://doi.org/10.1108/ s0193-2306(2012)0000015012

Leckie, G. y Charlton, C. (2012). Runmlwin: A program to run the MlwinN Multilevel Modeling Software from within Stata. Journal of Statistical Software, 52(11). https://doi. org/10.18637/jss.v052.i11

Leff, N.H. (1964). Economic development through bureaucratic corruption. American Behavioral Scientist, 8(3), 8-14. https://doi. org/10.1177/000276426400800303

Leys, C. (1965). What is the problem about corruption? Journal of Modern African Studies, 3(2), 215-230. https://doi.org/10.1017/ $\underline{\text { s0022278x00023636 }}$

Mauro, P. (1995). Corruption and Growth. The Quarterly Journal of Economics, 110(3), 681-712. https://doi.org/10.2307/2946696

Méon, P-G. y Sekkat, K. (2005). Does corruption grease or sand the wheels of growth? Public Choice, 122, 69-97. https://doi.org/10.1007/ s11127-005-3988-0

Miller, S. (2010). Institutional Corruption. En S. Miller, The Moral Foundations of Social Institutions: A Philosophical Study (pp. 154-175). New York: Cambridge University Press.

Mudrack, P.E. (1993). An investigation into the acceptability of workplace behaviors of a dubious ethical nature. Journal of Business Ethics, 12(7), 517-524.

https://doi.org/10.1007/bf00872373

Nichols, P.M. y Robertson, D.C. (Eds.) (2017). Thinking about Bribery Neuroscience, Moral Cognition and the Psychology of Bribery. Cambridge: Cambridge University Press. https://doi.org/10.1017/9781316450765

Pedersen, E.J., Kurzban, R. y McCullogh, M.E. (2013). Do humans really punish altruistically? A closer look. Proc. R. Soc. B 280(1758), 20122723.

https://doi.org/10.1098/rspb.2012.2723

Renner, E. (2004). Wie lässt sich Korruption wirksam bekämpfen? Vierteljahreshefte zur Wirtschaftsforschung, 73(2), 292-300. https:// doi.org/10.3790/vjh.73.2.292

Rose-Ackerman, S. (2006). Introduction and over- 
view. En Rose-Ackerman, S. (Ed.) International Handbook on the Economics of Corruption ( $p$. 14-38). Cheltenham, UK, Northampton, MA, USA: Edward Elgar Publishing.

Schulze, G.G. y Frank, B. (2003). Deterrence versus intrinsic motivation: experimental evidence on the determinants of corruptibility. Economics of Governance, 4(2), 143-160. https://doi.org/10.1007/s101010200059

Selten, R. (1967). Die Strategiemethode zur Erforschung des eingeschränkt rationalen Verhaltens im Rahmen eines Oligopolexperiments. En Sauermann, H. (Ed.) Beiträge zur experimentellen Wirtschafts-forschung (p. 136-168). Tübingen: J.C.B. Mohr (Paul Siebeck).

Shah D.V., Domke, D. y Wackman, D. (1996). "To Thine Own Self Be True": Values, Framing, and Voter Decision-Making Strategies. Communication Research, 23(5), 509-560. https://doi. org/10.1177/009365096023005001

Shah D.V., Domke, D. y Wackman, D. (2001). The Effects of Value-Framing on Political Judg- ment and Reasoning. En Framing Public Life, edited by S.D. Reese, O.H Gandy Jr, and A. Grant, (p. 227-244). Mahwah, NJ: Lawrence Erlbaum Associates.

Snijders, T.A.B. y Bosker, R.J. (1999) Multilevel Analysis: An Introduction to Basic and Advanced Multilevel Modeling. Thousand Oaks, CA: Sage.

Spitzer, M., Fischbacher, U., Herrnberger, B., Grön, G. y Fehr, E. (2007). The neural signature of social norm compliance. Neuron, 56(1), 185-196.

https://doi.org/10.1016/j.neuron.2007.09.011

Wirtz, J. y Kum, D. (2004). Consumer cheating on service guarantees. Journal of the Academy of Marketing Science, 32(2), 159-175.

https://doi.org/10.1177/0092070303261416

Zizzo, D.J. (2010). Experimenter demand effects in economic experiments. Experimental Economics, 13(1), 75-98. https://doi.org/10.1007/ $\underline{\mathrm{s} 10683-009-9230-\mathrm{Z}}$

ecibido: 23 de mayo de 2018 Aceptado: 7 de mayo de 2020 
